\title{
EMBOLISMO CEREBRAL DURANTE VUELO COMERCIAL: REPORTE DE CASO
}

\author{
David J. Orccosupa-Quispe1,a, Fiorella D. Mendoza-Cabrera1,a, Margot Mejía-Hurtado 2,b
}

\begin{abstract}
RESUMEN
Presentamos el caso de un varón de 25 años con antecedentes de consumo de marihuana y tabaco, que durante viaje en vuelo comercial a Cusco presentó disnea, pérdida de conciencia y estado epiléptico. Arribó a esta ciudad presentando hipotensión arterial, murmullo pulmonar abolido, sin recuperación de conciencia. La tomografía de tórax reveló bullas pulmonares y la tomografía mostró neumoencéfalo, diagnosticándose embolia gaseosa cerebral. La hipoxemia asociada a convulsiones y pérdida de conciencia en una persona joven durante el vuelo no es un evento común. La pérdida de la presión en la cabina durante el ascenso parece ser el evento desencadenante en pacientes con enfermedad pulmonar.
\end{abstract}

Palabras clave: Barotrauma; Embolia aérea; Accidente cerebrovascular; Viaje en avión (fuente: DeCS BIREME).

\section{CEREBRAL EMBOLISM DURING COMMERCIAL FLIGHT: CASE REPORT}

\begin{abstract}
We present the case of a 25-year old man with a history of marijuana and tobacco consumption who, during a commercial flight to Cusco, presented dyspnea, loss of consciousness, and epileptic condition. He arrived in this city presenting arterial hypotension, abolished pulmonary murmur, with no recovery of consciousness. The thorax tomography revealed lung bullae and the tomography showed pneumocephalus. He was diagnosed with cerebral gas embolism. Hypoxemia associated with seizures and loss of consciousness in a young person during a flight is not a common event. Loss of cabin pressure during climb appears to be the triggering event in patients with lung disease.
\end{abstract}

Keywords: Barotrauma; Air embolism; Stroke; Air travelling (source: MeSH NLM).

\section{INTRODUCCIÓN}

Las emergencias médicas durante los vuelos comerciales son inusuales, se estima que ocurren 16 emergencias médicas por millón de pasajeros y uno por cada 604 vuelos ${ }^{(1)}$. La mayoría de las emergencias médicas en vuelo se relacionan con síncope, síntomas respiratorios o síntomas gastrointestinales ${ }^{(1)}$. Al año ocurren 44 mil emergencias médicas aéreas; sin embargo, existe una falta de informes sobre estos eventos lo que dificulta las investigaciones en esta área ${ }^{(2)}$. La embolia gaseosa cerebral en vuelos aéreos es una emergencia poco frecuente, se han reportado sólo 18 casos hasta el $2017^{(3)}$.

La bulla gigante se define como aquella que ocupa el $30 \%$ del hemitórax, mayormente a consecuencia del tabaquismo, aunque pueden ser idiopáticas o tener otras causas ${ }^{(4-6)}$. El consumo concomitante de marihuana se ha asociado con bullas gigantes ${ }^{(5,7)}$. La prevalencia de bullas pulmonares es desconocida, aunque se describe que típicamente ocurre en los lóbulos superiores y es más frecuente en los varones ${ }^{(4)}$.

Nuestro objetivo es presentar un reporte de caso de embolia cerebral gaseosa fatal secundaria a la presencia de bullas pulmonares en un consumidor de marihuana.

\section{REPORTE DE CASO}

Presentamos el caso de un varón de 25 años residente de Lima, con antecedente de consumo de marihuana y tabaco, alérgico a los antinflamatorios no esteroideos y a penicilina, sin patologías conocidas, que realizó un viaje en un vuelo comercial de Lima a Cusco.

\footnotetext{
Asociación Científica de Estudiantes de Medicina Humana de la Universidad Andina del Cusco (ACIEMH-Andina), Universidad Andina del Cusco. Cusco, Perú. Departamento de Emergencia, Hospital Nacional Adolfo Guevara Velasco. Cusco, Perú.

Estudiante de Medicina; ${ }^{\mathrm{b}}$ médico internista

Recibido: 09/01/2019 Aprobado: 18/09/2019 En línea: 06/11/2019
}

Citar como: Orccosupa-Quispe DJ, Mendoza-Cabrera FD, Mejía-Hurtado M. Embolismo cerebral durante vuelo comercial: reporte de caso. Rev Peru Med Exp Salud Publica. 2019;36(4):705-8. doi: 10.17843/rpmesp.2019.360.4179 
A los 30 minutos iniciado el vuelo presentó de forma súbita disnea, convulsiones tónico-clónicas generalizadas sin recuperación de conciencia posictal. Durante el evento el paciente recibió medidas de atención paramédica. A su llegada a Cusco fue trasladado en una ambulancia a una clínica privada, sufriendo una nueva convulsión catalogándose como status convulsivo.

Ingresó a la Unidad de Trauma Shock con presión arterial de $60 / 30 \mathrm{mmHg}$, saturación de oxígeno de $81 \%$ con fracción inspiratoria de oxígeno de $40 \%$, frecuencia cardiaca de 56 latidos por minuto y frecuencia respiratoria de 36 respiraciones por minuto. Al examen físico se halló ingurgitación yugular bilateral, uso de músculos accesorios, tiraje intercostal y subclavicular, amplexación disminuida de tórax, a la percusión se encontró timpanismo en ambos campos pulmonares a predominio de bases, murmullo vesicular abolido en hemitórax derecho y disminuido en hemitórax izquierdo. En el examen cardiovascular se encontró ruidos cardiacos hipofonéticos, rítmicos y sin soplos, llenado capilar mayor a seis segundos. En el examen neurológico, el paciente no respondió al interrogatorio, la escala de Glasgow fue de cinco puntos, con pupilas isocóricas de $5 \mathrm{~mm}$, no reactivas, reflejo consensual abolido y reflejo corneal disminuido.

El análisis de gases arteriales mostró una $\mathrm{PaO}_{2}$ de $45 \mathrm{mmHg}$ con $\mathrm{FiO}_{2}$ de $40 \%$, con un cociente $\mathrm{PaO}_{2} / \mathrm{FiO}_{2}$ de 112,5, pH de $7,36, \mathrm{HCO}_{3}$ de $20 \mathrm{mmol} / \mathrm{L}, \mathrm{PCO}_{2}$ de $35 \mathrm{mmHg}$, gradiente alvéolo arterial de oxígeno de $116,45 \mathrm{mmHg}$, que fue indicativo de hipoxemia y acidosis metabólica. Los demás resultados de laboratorio fueron normales.

Se solicitó radiografía de tórax de proyección anteroposterior que mostró radiolucidez en ambos tercios inferiores con desplazamiento izquierdo de mediastino (Figura 1). En la Tomografía Espiral Multicorte (TEM) torácica sin contraste se evidenciaron imágenes que disminuyen la atenuación de paredes delgadas, sugestivas de bullas pulmonares
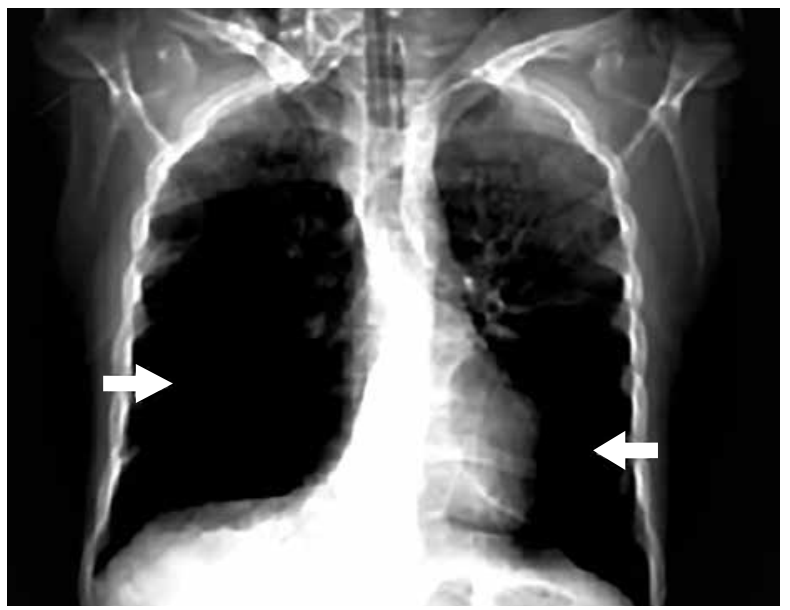

Figura 1. Radiografía de tórax con radiolucidez en ambos campos pulmonares (flechas blancas) y desplazamiento izquierdo de mediastino gigantes en ambos campos pulmonares (Figura 2) y la TEM cerebral sin contraste mostró edema cerebral difuso con pérdida de la diferenciación cortico-subcortical, ausencia de cisternas basales e imagen hipodensa sugestiva de neumoencéfalo (Figura 3).

Los diagnósticos finales fueron: bullas gigantes pulmonares bilaterales, neumoencéfalo por embolismo gaseoso de origen pulmonar, insuficiencia respiratoria aguda severa, shock obstructivo, status convulsivo y encefalopatía hipóxica isquémica global difusa.

La estabilización inicial se realizó en una clínica privada para posteriormente ser derivado al Hospital Nacional Adolfo Guevara Velasco del seguro social de Cusco. Se colocó un tubo orotraqueal conectado a ventilación mecánica, soporte vasopresor e inotrópico con noradrenalina y dopamina, sedoanalgesia, catéter venoso central infraclavicular derecho, dos cánulas de descompresión en tercer y quinto espacio intercostal del hemitórax derecho.

El análisis de gases arteriales de control mostró un $\mathrm{PaO}_{2}$ de $107,5 \mathrm{mmHg}$ con $\mathrm{FiO}_{2}$ de $100 \%$, con un cociente $\mathrm{PaO}_{2} / \mathrm{FiO}_{2}$ de 107,5, $\mathrm{PCO}_{2}$ de $39 \mathrm{mmHg}, \mathrm{pH}$ de 7,33, $\mathrm{HCO}_{3}$ de $20 \mathrm{mmol} / \mathrm{L}$, gradiente alvéolo arterial de oxígeno de $356,3 \mathrm{mmHg}$, mostrando acidosis metabólica. En la evolución se evidenció hemodinamia estable con soporte vasopresor. Al examen neurológico se evaluó escala RASS (Richmond Agitation-Sedation Scale) con puntaje de -4, pupilas midriáticas de $12 \mathrm{~mm}$ no reactivas, ausencia de reflejo corneal y tusígeno. El Servicio de Neurocirugía describió una evolución con criterios clínicos próximos de muerte cerebral no candidato de tratamiento neuroquirúrgico. Por tal motivo, el Servicio de Cirugía de Tórax y Cardiovascular decidió no realizar toracotomía derecha más bullectomía pulmonar. Finalmente, el paciente presentó parada cardiaca sin respuesta a la reanimación avanzada y falleció a las 72 horas de la primera convulsión, no se le realizó la necropsia por pedido del familiar.

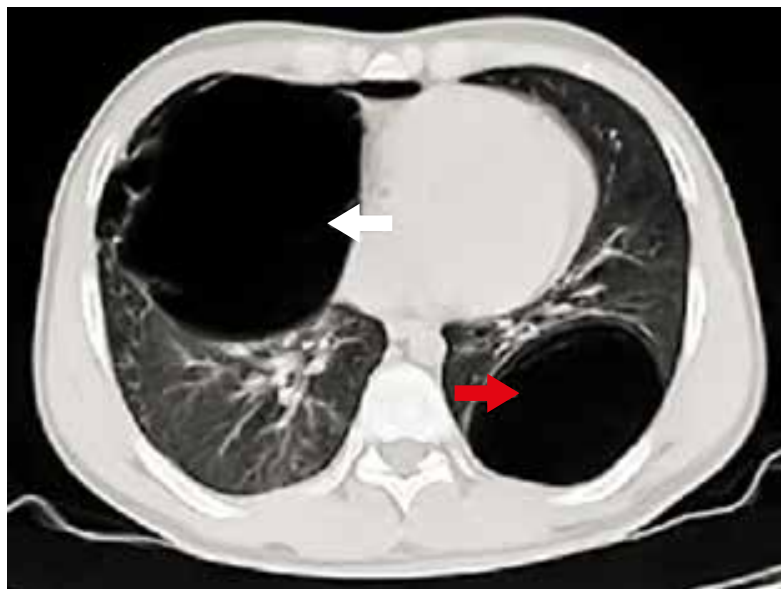

Figura 2. Tomografía Espiral Multicorte con bulla pulmonar derecha (flecha blanca) e izquierda (flecha roja) de $113,9 \mathrm{~cm} x$ $12 \mathrm{~cm} \times 17,1 \mathrm{~cm}$ y de $6,6 \mathrm{~cm} \times 7,8 \mathrm{~cm} \times 7,9 \mathrm{~cm}$, respectivamente 


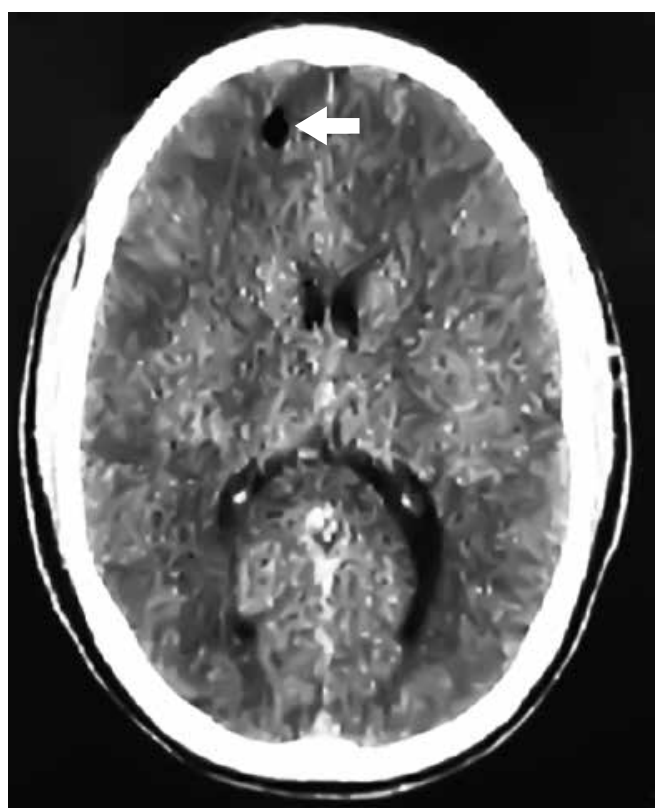

Figura 3. Tomografía Espiral Multicorte muestra edema cerebral difuso e imagen hipodensa sugerente de neumoencéfalo (flecha blanca)

\section{DISCUSIÓN}

Se ha descrito el síndrome del pulmón evanescente (Vanishing Lung Syndrome), el cual es una enfermedad rara que causa bullas pulmonares gigantes y ocupa al menos un tercio del hemitórax; usualmente se produce por fumar tabaco, aunque se han reportado casos que relacionan a la marihuana con su desarrollo e incluso como un catalizador potencial ${ }^{(5,8)}$. El paciente tenía antecedentes de estos hábitos nocivos.

El barotrauma pulmonar, por el cambio de presión durante el ascenso, produciría un embolismo gaseoso en un paciente con bullas pulmonares, lo cual es una situación no común $(6,9,10)$. La ley de Boyle-Mariotte determina que el volumen de un gas es inversamente proporcional a la presión a la que está sometido. Por consiguiente, cuando disminuye la presión barométrica en la cabina del avión durante el ascenso, se expandirá el aire atrapado dentro de una bulla pulmonar. A nivel del mar, la presión atmosférica es de alrededor $760 \mathrm{mmHg}$. Una cabina presurizada a 8000 pies tendrá una presión de alrededor de $35 \%$ a $40 \%$ menos que la presión atmosférica, lo que significa que habrá un aumento resultante en el volumen de gas de $35 \%$ a $40 \%$ en una cavidad no comunicante ${ }^{(11)}$. El incremento del volumen de una bulla pulmonar ocasiona un estiramiento de sus paredes con posterior rotura de éstas y liberación de su gas. El aire viaja a la circulación sistémica por tres mecanismos ya descritos en la literatura, como son: embolización directa de la burbuja de aire a las venas pulmonares; al sistema arterial pulmonar; y paradójicamente, a través de shunt funcional de derecha a izquierda ${ }^{(12)}$.
El embolismo cerebral posiblemente provoca una alteración en la barrera hematoencefálica, isquemia local y edema, lo que genera signos característicos de inicio súbito; en cambio, los embolismos en músculos o vísceras pueden no tener síntomas ${ }^{(13)}$. La distribución de las burbujas de aire en el cuerpo se ve influenciada por el flujo sanguíneo y su flotabilidad, siendo esta última la que más favorece la afectación cerebral en un paciente erguido o sentado ${ }^{(6,14)}$. Los signos neurológicos que el paciente presentó, debido al embolismo cerebral, iniciaron con pérdida de conciencia y con convulsiones tónica-clónicas; y como se describe en la literatura, estos síntomas comúnmente se dan al inicio del vuelo $(3,6,9,10)$.

El tratamiento generalmente consiste en la administración de oxígeno y uso de cámara hiperbárica bajo el sustento de la ley de Boyle-Mariotte que puede contrarrestar los efectos del neumoencéfalo ${ }^{(15)}$; sin embargo, la inestabilidad hemodinámica y ventilatoria de nuestro paciente lo impedían. Se describe a la bullectomía como indicación terapéutica en estos casos ${ }^{(8)}$, sin embargo, nuestro paciente no fue tributario de esta cirugía debido a la evolución clínica neurológica con reflejos corneal, tusígeno y nauseoso ausentes, además de la encefalopatía hipóxica isquémica global difusa, cuadros que no favorecían el pronóstico.

Como limitaciones del presente reporte debemos reconocer que realizamos una anamnesis indirecta debido a la condición crítica del paciente, sin poder determinar con más detalle los hábitos nocivos y síntomas previos al evento.

La incidencia y las características de las emergencias aéreas en Perú no son conocidas, se sugiere ampliar información sobre este tema a través de la creación de un registro oficial.

Los pacientes, especialmente si tienen factores de riesgo, que presenten signos de una posible bulla pulmonar deben ser diagnosticados y tratados oportunamente. Los profesionales de la salud deberían tomar en cuenta esta entidad en pacientes consumidores de tabaco y marihuana.

Finalmente, no informar al paciente con bullas pulmonares, especialmente si éstas son gigantes, sobre el riesgo de realizar un viaje aéreo y sus posibles complicaciones (como una embolia gaseosa) debido a los cambios de presión podría tener un desenlace fatal, a pesar de que estos eventos son excepcionales.

Agradecimientos: Al Dr. Charles Augusto Huamani Saldaña por la revisión crítica del artículo científico.

Contribuciones de autoría: DJOQ, FDMC, MMH han participado en la concepción, elaboración y diseño del artículo, además de recolección y obtención de los datos, redacción y revisión crítica del artículo. 
Fuentes de financiamiento: La presente investigación ha sido autofinanciada.

Conflictos de interés: Los autores declaran no presentar conflictos de interés.
Criterios éticos: Se obtuvo el consentimiento informado de la pareja del paciente. El permiso institucional del Hospital Nacional Adolfo Guevara Velasco fue otorgado previo a la aprobación del comité de ética para la publicación del reporte de caso.

\section{REFERENCIAS BIBLIOGRÁFICAS}

1. Peterson DC, Martin-Gill C, Guyette FX, Tobias AZ, McCarthy CE, HarringtonST, et al. Outcomes of medical emergencies on commercial airline flights. N Engl J Med. 2013;368(22):2075-83. doi: 10.1056/ NEJMoa1212052.

2. Sul C, Badawy SM. A Systematic Review of Pediatric and Adult In-Flight Medical Emergencies. Int J Pediatr. 2018.6596490. doi: 10.1155/2018/6596490.

3. Oyanguren B, Alonso-Cánovas A, de Felipe A,MasjuánJ.Cerebral AirEmbolism in Commercial Flights: A Potentially Fatal Complication of Intrathoracic Lesions. Int J Crit Care Emerg Med. 2017;3:022. doi: $10.23937 / 2474-3674 / 1510022$.

4. Gonzáles P, Hernández J, Fumero S. Evolución de una bulla gigante. Med Gen Fam. 2018;7(2):66-9. doi: 10.24038/mgyf.2018.017.

5. Wiesel S, Siddiqui F, Khan T, Hossri S, El-Sayegh D. Vanishing Lung Syndrome: Compound Effect of Tobacco and Marijuana Use on the Development of Bullous Lung Disease-A Joint Effort. Cureus. 2017;9(7):e1530. doi: 10.7759/ cureus. 1530.
6. Gudmundsdottir JF, Geirsson A Hannesson P, Gudbjartsson T. Major ischaemic stroke caused by an air embolism from a ruptured giant pulmonary bulla. BMJ Case Rep. 2015;2015. pii: bcr2014208159. doi: 10.1136/bcr-2014 208159.

7. JohnsonMK,Smith RP,MorrisonD, Laszlo G, White RJ. Large lung bullae in marijuana smokers. Thorax. 2000;55(4):340-2. doi: 10.1136/thorax.55.4.340.

8. Davies P, Bradley C. Vanishing lung syndrome: giant bullous emphysema. Lancet. 2017;390(10112):2583. doi: 10.1016/S0140-6736(17)32246-8.

9. SalamehJ.A62-year-oldwomanwithcerebral artery air embolism during commercial air travel. Neurologist. 2010;16(2):136. doi: 10.1097/NRL.0b013e3181cf8695.

10. Yeung JTH, Ma JKF, Mak YF, Lam VSC. Fatal cerebral air embolism related to an air flight. Hong Kong Med J. 2013;19(4):352-3. doi: $10.12809 /$ hkmj133898.

11. Gong H. Advising pulmonary patients about commercial air travel. Ann Intern Med. 1989;111(5):349-51. doi: 10.7326/0003-4819-111-5-349.
12. Dudney TM, Elliott CG. Pulmonary embolism from amniotic fluid, fat, and air. Prog Cardiovasc Dis. 1994;36(6):447-74.

13. Arnaiz J, Lucas EM de, Piedra T, Garcia MEA, Patel AD, Gutierrez A. In-flight Seizures and Fatal Air Embolism: The Importance of a Chest Radiograph. Arch Neurol. 2011;68(5):661-4. doi: 10.1001/archneurol.2011.85.

14. Van Allen CM, Hrdina LA, Clark J. Air embolism from the pulmonary vein-A clinical and experimental study. Arch Surg. 1929;19: 567-99. doi:10.1001/ archsurg.1929.01150040003001

15. Farshchi Zarabi S, Parotto M, Katznelson R, Downar J. Massive Ischemic Stroke Due to Pulmonary Barotrauma and Cerebral Artery Air Embolism During Commercial Air Travel. Am J Case Rep. 2017;18:660. doi: 10.12659/ajcr.903354.

Correspondencia: David Jonathan Orccosupa Quispe.

Dirección: Avenida de la Cultura 1210. Cusco, Perú.

Teléfono: 519842533111

Correoelectrónico:bresukite@hotmail.com 